\title{
Estudo da Influência dos Iniciadores na Polimerização da 2-Fenil-2-0xazolina
}

\author{
Pedro Ivo C. Guimarães \\ Instituto de Química - Grupo de Polímeros (GPol), Universidade do Estado do Rio de Janeiro
}

\begin{abstract}
Resumo: 2-Fenil-2-oxazolina foi polimerizada utilizando-se iodeto de metila, tosilato de metila, eterato de trifluoreto de boro e sulfatos de dimetila, dietila e di-sec-butila como iniciadores. Este trabalho visa estudar a influência do tipo de iniciador nos processos de iniciação e propagação bem como as condições reacionais dessas polimerizações. Os resultados das polimerizações mostraram que a nucleofilicidade do iniciador e os diferentes grupos alquilas presentes nos sulfatos de dialquila afetam a reação de iniciação e influenciam a propagação das espécies ativas devido ao efeito do contra-íon derivado desses iniciadores. Os sulfatos de dialquila investigados exibiram uma boa eficiência na iniciação e seus comportamentos foram próximos.
\end{abstract}

Palavras-Chave: Polimerização catiônica, poli(N-acil-etilenoiminas), iniciadores catiônicos, oxazolinas-2substituídas, heterocíclicos.

\section{Effect study of initiators in 2-phenyl-2-oxazoline polimerization}

Abstract: 2-Phenyl-2-oxazoline was polymerized using methyl iodide, methyl tosilate, etherate boron trifluorated, dimethyl, diethyl and di-sec-butyl sulphate as initiators. The main aim of this work was to study the influence of type of initiator on the initiation and propagation processes as well the reactional conditions of these polymerizations. The polymerizations results showed that the nucleophilicity of initiator and different alkyl groups present in the dialkyl sulphates affect the initiation reaction, and influence the propagation species due to the counteranion effect, derived from these initiators. The dialkyl sulphates investigated exhibited good initiator efficiency and their performances were close.

Keywords: Cationic polymerization, poly( $N$-acyl-ethyleneimine), cationic initiators, 2-substituted-oxazolines, heterocycles

\section{Introdução}

As poli(N-acil-etilenoiminas) são polímeros versáteis, obtidos a partir da polimerização de 2-alquil ou 2aril-2-oxazolinas. Os homo e copolímeros obtidos a partir de 2-oxazolinas apresentam diversar aplicações, tais como: surfactantes e hidrogéis não iônicos, polímeros em rede e reativos, agentes compatibilizantes, adesivos, estabilizadores poliméricos, catlisadores de transferência de fase, retardantes de chama e corantes têxteis, além de poderem ser empregados na separação cromatográfica de misturas racêmicas ou como materiais compatíveis com biopolímeros ${ }^{[1]}$.
A polimerização por abertura de anel das 2-oxazolinas é induzida por iniciadores catiônicos e não ocorre com iniciadores aniônicos ou radicalares. A 2-fenil-2-oxazolina é um ciclo-imino-éter que polimeriza por abertura de anel, entre as posições 1 e 5 , pela ação de iniciadores protônicos ou não-protônicos (I), produzindo a poli(N-benzoil-etilenoimina) (PBEI) Figura 1

Um dos aspectos importantes a ser avaliado nessa polimerização é o tipo de sistema iniciador empregado. O iniciador afeta significativamente a polimerização das 2-oxazolinas e em alguns casos são produzidos polímeros com grupos terminais reativos, que podem ser posteriormente utilizados.

Autor para correspondência: Pedro Ivo Canesso Guimarães, Universidade do Estado do Rio de Janeiro, Instituto de Química, Grupo de Polímeros (GPol), Rua São Francisco Xavier, 524, Pavilhão Reitor Haroldo Lisboa da Cunha, sala 406, Maracanã, CEP: 20559-900, Rio de Janeiro/RJ. E-mail:canesso@uerj.br 


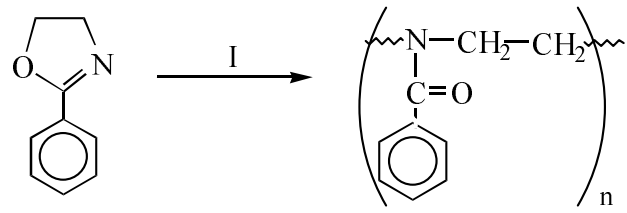

Figura 1 - Síntese da poli(N-benzoil-etilenoimina)

As 2-oxazolinas possuem elevada basicidade quando comparadas a outros monômeros que também polimerizam cationicamente. Geralmente, destroem os iniciadores tipo ácido de Lewis. Entretanto, os ácidos oxigenados e seus ésteres bem como haletos de alquila são iniciadores efetivos para estes monômeros ${ }^{[2]}$.

Em trabalhos anteriores, Kagiya e Matsuda estudaram a polimerização de complexos de 2-fenil2-oxazolina com ácidos de Brönsted, investigaram a eficiência destes iniciadores e propuseram mecanismos para esta polimerização ${ }^{[3]}$. Segundo esses autores, os sais oxazolínicos de ácido perclórico e p-toluenossulfônico exibem alta atividade catalítica, enquanto que os sais de $\mathrm{HCl}, \mathrm{HNO}_{3}$ e ácido trifluoroacético não são capazes de iniciar a polimerização. O sulfato de dimetila também foi descrito como um bom iniciador para este monômero, produzindo um polímero com uma elevada viscosidade intrínseca ${ }^{[4]}$. Bartz e colaboradores polimerizaram 2-metil-2-oxazolina e 2-fenil-2-oxazolina utilizando como iniciadores 9-bromo-metil-antraceno e 9-(3-bromo-propil)-antraceno, o que permitiu introduzir o antraceno como grupo terminal da polioxazolina formada ${ }^{[1,5]}$. Cloroformatos polifuncionais, como o tris-(cloroformato) de 2,2-bis(hidroxi-metil)-1-butanol foram empregados como iniciadores para a polimerização de 2-fenil-2oxazolina obtendo-se polímeros estrela de três ra$\operatorname{mos}^{[1,6]}$. Copolímeros em bloco desse monômero ou 2-metil-2-oxazolina e poli(glicóis etilênicos) com diferentes pesos moleculares e contendo grupos terminais cloroformatos também foram sintetizados com bons resultados ${ }^{[1,6]}$. Copolímeros em bloco de 2-metil e 2-fenil-2-oxazolinas foram produzidos utilizandose derivados bicíclicos da N-acetil-D-glicosamina como iniciadores ${ }^{[1,7]}$. Apesar dos estudos já realizados, pouco se averigou sobre o efeito do tamanho do grupo alquila e da nucleofilicidade dos iniciadores nestas polimerizações catiônicas.

Neste trabalho realizou-se o estudo da influência do tipo de iniciador na polimerização catiônica em massa da 2-fenil-2-oxazolina, com o objetivo de verificar a influência da nucleofilicidade e do tamanho do grupo alquila presente no iniciador. Foram empregados como iniciadores três diferentes sulfatos de dialquila (sulfato de dimetila, sulfato de dietila, sulfato de di-sec-butila), além do tosilato de metila, iodeto de metila e eterato de trifluoreto de boro.

\section{Experimental}

Materiais: A 2-fenil-2-oxazolina foi sintetizada e purificada de acordo com a literatura ${ }^{[8]}$. O sulfato de di-sec-butila foi obtido segundo o método desenvolvido por Katsuno ${ }^{[8,9]}$, enquanto o tosilato de metila foi preparado através de uma síntese em duas etapas $^{[10]}$. O sulfato de dimetila (Vetec), sulfato de dietila (Merck), iodeto de metila (Merck) foram destilados por duas vezes à pressão reduzida e o eterato de trifluoreto de boro (BDH) foi usado como recebido. Outros reagentes e solventes foram purificados quando necessário pelos métodos usuais.

Método: Em tubos de vidro (sistemas de polimerização) adicionou-se $1,0 \mathrm{~mL}$ do monômero e iniciador variando-se a razão molar entre eles (Tabela 1). Foram utilizados como iniciadores o sulfato de dimetila (SDMe), sulfato de dietila (SDEt), sulfato de di-sec-butila (SDsBu), tosilato de metila (TsOMe), iodeto de metila (IMe) e o eterato de trifluoreto de boro (TFBE). Os sistemas foram deaerados com nitrogênio seco, selados e as polimerizações realizadas em diferentes tempos reacionais (Tabela 2) e temperaturas (Tabela 3). Ao término das polimerizações, os produtos foram solubilizados em $10 \mathrm{~mL}$ de clorofórmio e precipitados em excesso de éter etílico, sucessivas vezes. Os polímeros obtidos foram filtrados e secos sob vácuo. Cada polimerização foi realizada em duplicata. O monômero, os iniciadores e polímeros foram caracterizados por FTIR (FTIR Perkin-Elmer 1720X), apresentando bandas correspondentes as citadas na literatura ${ }^{[8]}$.

\section{Resultados e Discussão}

Inicialmente, foram realizados ensaios preliminares onde polimerizou-se 2-fenil-2-oxazolina em diferentes razões molares monômero/iniciador, tempos reacionais e temperaturas, a fim de se determinar as melhores condições reacionais para a obtenção das conversões mais elevadas para o sistema em estudo. 
Tabela 1. Influência da razão molar monômero/iniciador na polimerização da 2-fenil-2-oxazolina

\begin{tabular}{|c|c|c|c|c|c|c|c|}
\hline \multirow[t]{2}{*}{ Iniciador } & \multicolumn{7}{|c|}{ Monômero/iniciador / Rendimento da polimerização (\%) } \\
\hline & 25 & 50 & 100 & 250 & 500 & 1.000 & 2.500 \\
\hline SDMe & 97 & 100 & 93 & 100 & 14 & - & - \\
\hline SDEt & 100 & 100 & 100 & 100 & 100 & 100 & - \\
\hline $\mathrm{SDsBu}$ & 100 & 100 & 100 & 100 & 96 & - & - \\
\hline TsOMe & 100 & 100 & 100 & 100 & - & - & - \\
\hline TFBE & 100 & 92 & 100 & 100 & - & - & - \\
\hline $\mathrm{IMe}$ & 100 & 100 & 100 & 100 & 15 & - & - \\
\hline
\end{tabular}

Condições reacionais: temperatura: $130^{\circ} \mathrm{C}$; tempo: $48 \mathrm{~h}$

Os iniciadores foram completamente solúveis nos monômeros, formando assim sistemas homogêneos. A 2-fenil-2-oxazolina foi polimerizada, variando-se a razão molar monômero/iniciador entre 25 e 2.500 (Tabela 1).

Observou-se que as polimerizações não ocorreram quando maiores razões molares monômero/iniciador foram empregadas, exceto quando SDEt foi usado. Segundo Tanaka e colaboradores, em razões monômero/iniciador muito elevadas, o grau de polimerização cai devido a influência de impurezas (principalmente as não voláteis). Como a maioria das polimerizações catiônicas, a de 2-fenil-2oxazolina é muito sensível a impurezas, o monômero não tratado, contendo água, deve ser polimerizado utilizando concentrações elevadas de iniciador. Entretanto obtêm-se produtos com baixas viscosidades (baixo peso molecular) ou ramificações ${ }^{[11]}$. Portanto, pode-se supor que em razões monômero/inicia- dor mais altas, as polimerizações não ocorrem devido à presença de contaminantes, no monômero e nos iniciadores. Nestas condições a quantidade de iniciador empregada é insuficiente para iniciar a polimerização, pois parte dele foi consumido pelas impurezas reativas.

A temperatura é um fator importante no processo de iniciação catiônica. Sua influência foi verificada entre $70^{\circ} \mathrm{C}$ e $170^{\circ} \mathrm{C}$ (Tabela 2).

A $70^{\circ} \mathrm{C}$ as polimerizações não ocorreram, provavelmente devido ao tempo reacional ter sido insuficiente (reação muito lenta) para se obter os polímeros nesta temperatura.

A dependência do tempo foi observada em intervalos de 12 horas por um período de 60 horas (Tabela 3).

$\mathrm{Na}$ Tabela 3, observa-se que as polimerizações tiveram elevados rendimentos nos diferentes tempos reacionais empregados.

Tabela 2. Influência da temperatura na polimerização da 2-fenil-2-oxazolina

\begin{tabular}{ccccccc}
\hline Iniciador & \multicolumn{5}{c}{ Temperatura/Rendimento da polimerização (\%) } \\
& $70^{\circ} \mathrm{C}$ & $90^{\circ} \mathrm{C}$ & $110^{\circ} \mathrm{C}$ & $130^{\circ} \mathrm{C}$ & $150^{\circ} \mathrm{C}$ & $170^{\circ} \mathrm{C}$ \\
\hline SDMe & - & 35 & 100 & 100 & 87 & 100 \\
SDEt & - & 97 & 100 & 100 & 100 & 100 \\
$\mathrm{SDsBu}$ & - & 79 & 92 & 100 & 100 & 95 \\
$\mathrm{TsOMe}$ & - & 90 & 100 & 100 & 96 & 100 \\
$\mathrm{TFBE}$ & - & 93 & 97 & 100 & 99 & 100 \\
$\mathrm{IMe}$ & - & - & 38 & 100 & 100 & 100 \\
\hline
\end{tabular}

Condições reacionais: tempo: $48 \mathrm{~h}$; razão molar monômero/iniciador $=250$ 
Tabela 3. Influência do tempo na polimerização da 2-fenil-2-oxazolina

\begin{tabular}{cccccc}
\hline Iniciador & \multicolumn{5}{c}{ Tempo/Rendimento da polimerização (\%) } \\
& $12 \mathrm{~h}$ & $24 \mathrm{~h}$ & $36 \mathrm{~h}$ & $48 \mathrm{~h}$ & $60 \mathrm{~h}$ \\
\hline SDMe & 90 & 100 & 91 & 100 & 100 \\
SDEt & 100 & 100 & 99 & 100 & 100 \\
$\mathrm{SDsBu}$ & 97 & 81 & 100 & 100 & 87 \\
$\mathrm{TsOMe}$ & 88 & 95 & 90 & 100 & 100 \\
$\mathrm{TFBE}$ & 98 & 85 & 94 & 100 & 92 \\
$\mathrm{IMe}$ & 61 & 100 & 100 & 100 & -
\end{tabular}

Condições reacionais: temperatura: $130^{\circ} \mathrm{C}$; razão molar monômero/ iniciador $=250$

$$
\begin{aligned}
& \text { 至 }
\end{aligned}
$$

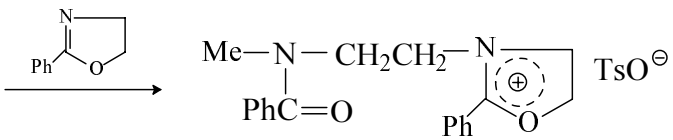

Figura 2. Iniciação da polimerização da 2-fenil-2-oxazolina pelo tosilato de metila

Os iniciadores apresentaram resultados muito próximos para a maioria das polimerizações, exceto para elevados valores de razão monômero/iniciador. Os melhores resultados (altas conversões) para as polimerizações da 2-fenil-2-oxazolina, com diferentes iniciadores, foram conseguidos na razão molar monômero/iniciador $=250$, a $130^{\circ} \mathrm{C}$ por 48 horas.

A natureza da espécie propagante na polimerização de uma 2-oxazolina é controlada pela nucleofilicidade relativa do monômero e do contraíon derivado do iniciador, bem como pelas condições reacionais $^{[12]}$.

A espécie propagante é formada pela interação de um iniciador, geralmente um reagente alquilante, com o centro nucleofílico (nitrogênio) do anel oxazolínico. A maioria das polimerizações das 2oxazolinas emprega iniciadores metilantes, tais como IMe, TsOMe e SDMe. O eletrófilo do iniciador é incorporado ao final da cadeia, sendo independente da natureza da extremidade propagante ${ }^{[13]}$.

Vários estudos mostraram que as polimerizações iniciadas por TsOMe têm como etapa determinante da velocidade de propagação, o processo de abertura do anel oxazolínico pelo ataque do monômero, formando uma espécie propagante iônica (Figura $2)^{[14-18]}$.

Por outro lado, na polimerização induzida por IMe, a velocidade é controlada pela formação do haleto oxazolínico instável que sofre abertura do anel heterocíclico e origina uma ligação covalente do haleto com o sal oxazolínico, sendo esta a espécie propagante.

Os sulfatos de dialquila possuem comportamento análogo ao TsOMe e formam íons estáveis com as 2oxazolinas.

A diferença de mecanismo entre estas polimerizações pode ser explicado pela diferença de reatividade nucleofílica entre os contra-íons. De acordo com Swain-Scott-Hime, a constante de nucleofilicidade (n) do iodeto $(\mathrm{n}=5,04)$ é muito maior que do tosilato $(\mathrm{n}<1,0)$ ou do sulfato de alquila. Um nucleófilo forte como o iodeto rapidamente abre o anel do sal oxazolínico produzindo a espécie covalente do iodeto com o sal de oxazolina, enquanto que nucleófilos fracos como o tosilato ou o sulfato de alquila não têm a capacidade de atacar a espécie propagante. Nesse último caso, a espécie iônica no final da cadeia é atacada sucessivamente pelo monômero, produzindo um polímero com elevado grau de polimerização ${ }^{[14]}$. Portanto, na polimerização iniciada por TsOMe ou sulfato de dialquila a 2-fenil2-oxazolina possui um caráter nucleofílico mais forte que os contra-íons dos iniciadores, enquanto que o
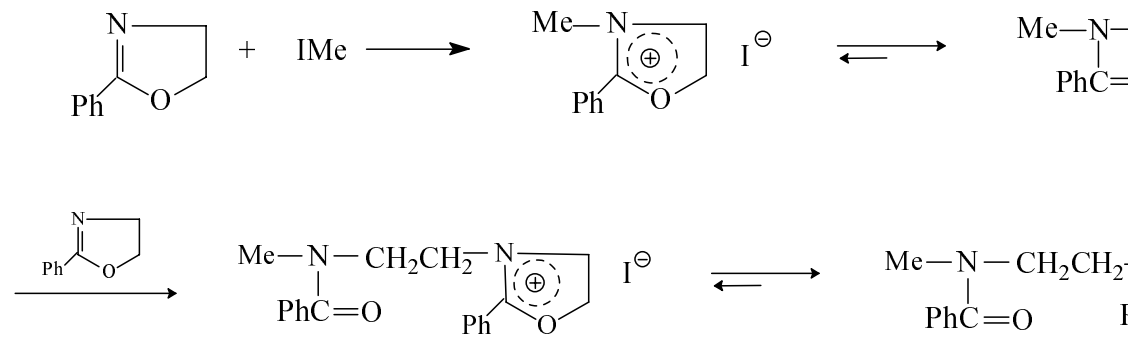

Figura 3. Iniciação da polimerização da 2-fenil-2-oxazolina pelo iodeto de metila 
monômero é um nucleófilo mais fraco em relação ao iodeto $^{[15]}$.

O caráter de íon livre da espécie propagante induz o aumento da velocidade de propagação ${ }^{[3]}$. Logo, as espécies propagantes iônicas, se estiverem dissociadas, tendem a polimerizar mais rapidamente que as espécies propagantes covalentes.

O TFBE é consumido quase que completamente nos primeiros estágios da polimerização, formando o sal oxazolínico pela complexação do ácido de Lewis com o monômero que propaga através do mecanismo iônico. Logo, o iniciador não gera um contra-íon livre, sendo o efeito deste contra-íon minimizado durante a propagação.

$\mathrm{Na}$ reação iniciada pelos sulfatos de dialquila, o sal oxazolínico é a espécie ativa no processo de propagação. Em geral, esta reação entre 2-oxazolina e o iniciador envolve um mecanismo de substituição nucleofílica bimolecular $\left(\mathrm{S}_{\mathrm{N}} 2\right)$, onde o anel oxazolínico é atacado pelo iniciador. Entretanto, é possível que o iniciador sofra uma dissociação heterolítica, formando um carbocátion (Figura 4).

A etapa determinante da velocidade neste processo é a dissociação do iniciador.

Quando iniciadores com grupos alquila secundários ou terciários são usados, a iniciação também pode ocorrer por um mecanismo de substituição nucleofílica unimolecular $\left(\mathrm{S}_{\mathrm{N}} 1\right)$.

Ingold examinou o efeito dos diferentes grupos alquila na velocidade e no mecanismo de substituições nucleofílicas de compostos alquílicos, onde o meio reacional e temperatura foram mantidos constantes. Este autor propôs uma série em que os grupos alquila estão arranjados em ordem crescente de capacidade de doar elétrons. No início desta série, o grupo metila sofre mecanismo $\mathrm{S}_{\mathrm{N}} 2$, mas ao longo da série, o mecanismo unimolecular torna-se predominante devido ao forte efeito cinético de liberar elétrons deste mecanismo ${ }^{[19,20]}$.

Os sulfatos secundários são mais susceptíveis à dissociação heterolítica do que os primários, segundo

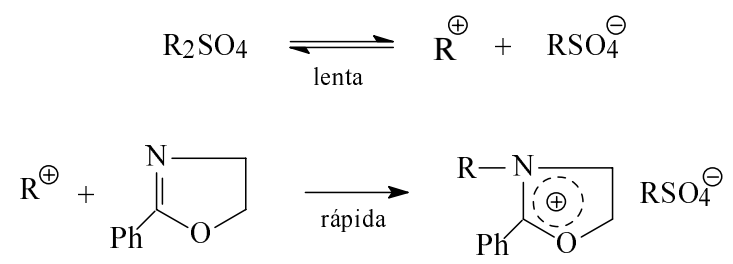

Figura 4. Dissociação heterolítica do sulfato de dialquila o observado em suas hidrólises ${ }^{[21]}$. Eles mostram características intermediárias, algumas vezes realizando mecanismo $\mathrm{S}_{\mathrm{N}} 2$, outras seguindo o mecanismo $\mathrm{S}_{\mathrm{N}} 1$ e, freqüentemente, uma mistura dos dois mecanismos. $\mathrm{O}$ tipo de mecanismo envolvido é determinado pela natureza e facilidade de dissociação do iniciador.

Dependendo do grupo alquila presente no iniciador, um contra-íon diferente é formado, afetando a reatividade da espécie propagante de modo desigual. Os sulfatos de alquila são bons grupamentos de saída, com elevada estabilidade devido ao efeito de ressonância do grupo sulfato; logo, os diferentes grupos alquilas presentes na estrutura do contra-íon podem afetar este efeito de ressonância. Os grupos alquila secundários podem favorecer o efeito de ressonância do grupo sulfato, reduzindo a nucleofilicidade do contra-íon.

Assim, segundo estes resultados e de acordo com a natureza da espécie propagante na polimerização de 2-fenil-2-oxazolina com os iniciadores empregados, a 2-fenil-2-oxazolina e os contra-íons derivados dos iniciadores podem ser arranjados na seguinte ordem decrescente de nucleofilicidade ${ }^{[10]}$ :

$$
\mathrm{I}^{-}>\text {2-fenil-2-oxazolina }>\mathrm{TsO}^{-}>\mathrm{RSO}_{4}^{-}
$$

Comparativamente, 2-fenil-2-oxazolina possui uma nucleofilicidade mais baixa que 2-metil-2oxazolina e 2-oxazolina não substituída devido ao efeito de ressonância do anel aromático conjugado ao anel heterocíclico.

Com base nestas observações, é razoável assumir que o tipo de iniciador bem como o grupo alquila presente nos sulfatos têm influência na reação de iniciação e um efeito indireto na reação de propagação da polimerização em massa de 2-fenil-2-oxazolina. A dissociação de sulfato de dialquila é dependente do grupo alquila presente em sua estrutura e da temperatura reacional.

\section{Conclusões}

De acordo com os resultados obtidos verificou-se que os iniciadores apresentaram resultados muito próximos para a maioria das polimerizações. Entretanto, a natureza da espécie propagante na polimerização de uma 2-oxazolina é controlada pela nucleofilicidade relativa do monômero e do contra-íon derivado do iniciador, o que indica que o tipo de iniciador afeta significativamente o desempenho da reação. 


\section{Referência Bibliográficas}

1. Aoi, K. \& Oada, M. - Prog. Polym. Sci., 21, p. 151 (1996).

2. Litt, M.; Levy, A. \& Herz, J. - J. Macromol. Sci., A-9, 5, p. 703 (1975).

3. Kagiya, T. \& Matsuda, T. - J. Macromol. Chem. Sci., A5, 8, p. 1265 (1971).

4. Bassiri, T. G.; Levy, A. \& Litt, M. - J. Polym. Sci., Part C: Polym. Lett. Ed., 5, p. 871 (1967).

5. Bartz, T.; Klaper, M. \& Mullen, K. - Macromol. Chem. Phys., 195, p. 1097 (1994).

6. Dworak, A. \& Schulz, R. C. - Makromol. Chem., 192, p. 437 (1991).

7. Aoi, K.; Suzuki, H. \& Okada, M. - Macromolecules, 25, p. 7073 (1992).

8. Guimarães, P. I. C.; Monteiro, A. P. \& Mazzei, J. L. Eur. Polym. J., 12, p. 1251 (1995).

9. Katsuno, M. - J. Soc. Chem. Ind. Japan, 44, p. 275 (1941).

10. Vogel, A . I. - "Química Orgânica - Análise Orgânica Qualitativa”, vol. 2, 2a edição, Ao Livro Técnico, Rio de Janeiro (1982).

11. Tanaka, R.; Ueoka, I.; Takake, Y. \& Tanaka, T. - J. Polym. Sci., Polym. Lett. Ed., 16, p. 13 (1976).
12. Saegusa, T. \& Kobayashi, S. - Macromolecular Science, in "International Review of Science", Physical Chemistry Series Two, vol. 8, Butterworth Publishers, London (1975).

13. Schultz, R. C. \& Schwarzenbach, E. - Makromol. Chem., Macromol. Symp. 13/14, p. 495 (1988).

14. Saegusa, T.; Kobayashi, S. \& Yamada, A. Makromol. Chem., 177, p. 2271 (1976).

15. Saegusa, T.; Ikeda, H. \& Fujii, H. - Macromolecules, 6, p. 315 (1973).

16. Saegusa, T. \& Ikeda, H. - Macromolecules, 6, p. 808 (1973).

17. Saegusa, T. - Makromol. Chem., 175, p. 1199 (1974).

18. Miyamoto, M.; Aoi, K. \& Saegusa, T. - Macromolecules, 21, p. 1880 (1988).

19. Morrison, R. T. \& Boyd, R. N. - "Organic Chemistry", $5^{\text {th }}$ edition, Allyn and Bacon, Newton (1987).

20. Ingold, C. K. - "Structure and Mechanism in Organic Chemistry", Cornell University Press, London (1969).

21. Mazzei, J. L. - "Cinética da Reação entre Sec-Butanol e Ácido Sulfúrico com uma Nova Abordágem Analítica", Tese de Mestrado, Universidade Federal do Rio de Janeiro, Brasil (1994)

Recebido: 03/08/00

Aprovado: 15/12/00 